\title{
The initial condition for long-term mantle evolution of rocky planets
}

\author{
MAXIM D. BALLMER $^{12}$, ROB SPAARGAREN ${ }^{2}$, \\ ANANYA MALLIK ${ }^{3}$, DANIELA BOLRÃO ${ }^{2}$, \\ ADRIEN MORISON ${ }^{4}$, MIKI NAKAJIMA $^{5}$
}

${ }^{1}$ Dept. Earth Sciences, Univ. College London,, UK. ${ }^{2}$ Inst. of Geophysics, ETH Zürich, Switzerland. ${ }^{3}$ Department of Geosciences, Univ. Rhode Island, USA. ${ }^{4}$ ENS Lyon;

UCBL, Univ. de Lyon, France. ${ }^{5}$ Univ. Rochester, USA

Terrestrial planets evolve through stages of large-scale melting, or magma oceans, due to the energy release during accretion and differentiation. Any magma ocean is thought to become progressively enriched in $\mathrm{FeO}$ and incompatible elements upon freezing due to fractional crystallization. The resulting upwards enrichment of the cumulate (=crystal) package(s) drives gravitational over-turn(s) of the incipient mantle, and ultimately stabilizes a $\mathrm{FeO}$-enriched molten layer at the core-mantle boundary (CMB), or "basal magma ocean" (BMO). The BMO itself is expected to freeze by fractional crystallization, ultimately stabilizing a thick FeO-enriched layer at the CMB. Such a layer, however, would be too dense to be entrained by mantle convection, a scenario that is ruled out by geophysical observations, at least for Earth.

Here, we investigate the consequences of an alternative mechanism, BMO reactive crystallization, on the initial condition of solid-state mantle convection and long-term planetary evolution. Any early BMO cumulates are readily entrained by mantle convection, and once the BMO-mantle boundary is exposed, the BMO reacts with the mantle to form reactive cumulates. Reaction is driven by disequilibrium between mantle rocks and the BMO. We show that entrainment of reactive cumulates is efficient such that reaction proceeds swiftly. For a wide range of BMO initial compositions, the final cumulate package consists of two discrete layers: the first is pure bridgmanite $\left(\sim \mathrm{MgSiO}_{3}\right)$; the second is similar to an enriched (in terms of $\mathrm{FeO}$ and incompatibles) pyrolite. The first layer is expected to be entrained by mantle convection due to its intrinsic buoyancy, but resist efficient mixing due to its intrinsic strength, thereby potentially providing an explanation for seismic scatterers/ reflectors and ancient geochemical reservoirs. The second layer is expected to stabilize thermochemical piles, providing a candidate origin for the large low shear velocity provinces.

These results have implications for the evolution of planets in general. Terrestrial planets that are too small to stabilize a BMO in their histories, such as Mars (?), may host a thicker and more strongly FeO-rich deep dense layer than Earth, which should efficiently slow down planetary cooling. 